\title{
An Optimization on Quadrature Formulas and Numerical Solutions of Ordinary Differential Equations
}

\author{
Venelin Todorov \\ Bulgarian Academy of Sciences \\ Institute of Mathematics and Informatics \\ Sofia 1113, Bulgaria \\ Bulgarian Academy of Sciences
}

Institute of Information and Communication Technologies

Sofia 1113, Bulgaria

Email: vtodorov@math.bas.bg,venelin@parallel.bas.bg

Radan Miryanov

University of Economics

Department of Statistics and Applied Mathematics

77 Knyaz Boris I Blvd.,

Varna 9002, Bulgaria

Email: miryanov@ue-varna.bg

Stefka Fidanova

Bulgarian Academy of Sciences

Institute of Information and Communication Technologies

ul. G. Bonchev 25A, 1113 Sofia, Bulgaria

Email: stefka@parallel.bas.bg

\author{
Yuri Dimitrov \\ Department of Mathematics and Physics \\ University of Forestry \\ Sofia 1756, Bulgaria \\ Bulgarian Academy of Sciences \\ Institute of Mathematics and Informatics \\ Sofia 1113, Bulgaria \\ Email: yuri.dimitrov@1tu.bg \\ Ivan Dimov \\ Bulgarian Academy of Sciences
}

Institute of Information and Communication Technologies

ul. G. Bonchev 25A, Sofia 1113, Bulgaria

Email: ivdimov@bas.bg

Stoyan Poryazov

Bulgarian Academy of Sciences

Institute of Mathematics and Informatics

ul. G. Bonchev 8, 1113 Sofia, Bulgaria

Email: stoyan@math.bas.bg

\begin{abstract}
This paper is a continuation of the discussion on optimization of the quadrature formulas and their applications in paper [2]. Second-order numerical solutions of Volterra integral equations are constructed using the quadrature formulas obtained in [2]. The numerical results presented in the paper confirm the effectiveness of the methods for numerical solution of ordinary differential equations.
\end{abstract}

\section{INTRODUCTION}

I $\mathrm{N}$ PAPER [2] we study the quadrature formulas which have generating functions $G_{1}(x)=\pi \sec (\pi \sqrt{x} / 2) / 4$ and $G_{2}(x)=\pi \tan (\pi \sqrt{x} / 2) /(4 \sqrt{x})$. We construct the secondorder quadrature formulas

$\frac{h}{2}\left(y_{0}+\sum_{k=1}^{N-1} \bar{E}_{k} y_{N-k}+\frac{\pi-1}{2} y_{N}\right)=\int_{a}^{b} y(x) d x+O\left(h^{2}\right)$,

Venelin Todorov is supported by the National Scientific Program "Information and Communication Technologies for a Single Digital Market in Science, Education and Security (ICT in SES)", contract No DO1-205/23.11.2018, financed by the Ministry of Education and Science in Bulgaria and by the Bulgarian National Science Fund under Project DN 12/5-2017 "Efficien Stochastic Methods and Algorithms for Large-Scale Problems". Yuri Dimitrov is supported by the Bulgarian National Science Fund under Young Scientist Project KP-06-M32/2 - 17.12.2019 "Advanced Stochastic and Deterministic Approaches for Large-Scale Problems of Computational Mathematics".
$\frac{h}{2}\left(y_{0}+\sum_{k=1}^{N-1} \bar{B}_{k} y_{N-k}+\frac{\pi^{2}-6}{4} y_{N}\right)=\int_{a}^{b} y(x) d x+O\left(h^{2}\right)$, where $h=(b-a) / N$ and $x_{k}=a+k h, y_{k}=y\left(x_{k}\right)$ for $k=0,1, \cdots, N$ and $\bar{E}_{k}$ and $\bar{B}_{k}$ are the coefficients of the Maclaurin series of the generating functions

$$
\bar{E}_{k}=\frac{\left|E_{2 k}\right|}{(2 k) !}\left(\frac{\pi}{2}\right)^{2 k+1}, \bar{B}_{k}=\frac{\left(4^{k+1}-1\right) \pi^{2 k+2}\left|B_{2 k+2}\right|}{(2 k+2) !},
$$

where $E_{k}$ and $B_{k}$ are the Euler and Bernoulli numbers. The coefficients of the right endpoint expansion formulas are equal to the coefficients of the Maclaurin series of the functions $H_{i}(x)=G_{i}\left(e^{-x}\right)$ for $i=1,2$. In [2] we construct third-order and fourth-order quadrature formulas as linear combinations of the trapezoidal rule

$$
\frac{h}{2}\left(y_{0}+2 \sum_{k=1}^{N-1} y_{N-k}+y_{N}\right)=\int_{a}^{b} y(x) d x+O\left(h^{2}\right)
$$

the two quadrature formulas above and their modifications. The method for construction of quadrature formulas by first specifying the generating function is an effective method for construction of approximations of the fractional derivatives and integrals (see [1], [3], [4], [5]). The method is applicable 
for construction of approximations of the definite integral and the integer order derivatives of a function as well. In [7] we construct approximations of the first derivative which have exponential and logarithmic generating functions. In the paper we give a proof for the convergence of the approximations and applications of the approximations for numerical solution of ordinary and partial differential equations. The method used in the paper can be extended for construction of approximations of the second derivative which are suitable for deriving approximations of the fractional derivatives. Other methods for numerical solution of integral equations use Gaussian quadratures on non-uniform nets and Monte Carlo methods for numerical integration (see. [8], [9], [10]). Let

$$
\frac{h}{2} \sum_{i=0}^{N} w_{i} y_{N-i}=\int_{a}^{b} y(t) d t+O\left(h^{2}\right)
$$

be a second-order quadrature. Consider a Voltera integral equation of the second kind which has the following form

$$
y(x)+\int_{0}^{x} K(x-t) y(t) d t=F(x) .
$$

The Nyström method (see [6]) for computing the numerical solution of equation (3) uses the approximations of the values of the definite integral in equation (3) with quadrature formula (*) on all intervals $\left[0, x_{n}\right]$,

$$
y_{n}+\frac{h}{2} \sum_{i=0}^{n} w_{i} K_{i} y_{n-i}=F_{n}+O\left(h^{2}\right) .
$$

The numerical solution $\left\{u_{n}\right\}_{n=0}^{N}$ of integral equation (3), where $u_{n}$ is an approximation of the value of the solution $y_{n}$, is computed recursively with

$$
u_{n}=\frac{1}{2+w_{0} K_{0} h}\left(2 F_{n}-h \sum_{i=1}^{n} w_{i} K_{i} y_{n-i}\right)
$$

and has an initial condition $u_{0}=y_{0}=F(0)$. The computation of numerical solution $\mathrm{NS}(*)$ involves $O\left(N^{2}\right)$ operations. Denote by (3.1) and (3.2) the equations obtained from equation (3) with a kernel function $K(s)=3+2 s$ and right hand side $F_{1}(x)=x^{3}\left(10\left(4 x^{2}+30 x+40\right) \ln x-18 x^{2}-75 x\right) / 400$ and

$$
\begin{aligned}
F_{2}(x)=\arctan x-x & +(3 / 2+x) \ln \left(1+x^{2}\right) \\
& +\left(1+3 x+x^{2}\right) \operatorname{arccot} x
\end{aligned}
$$

respectively. Equations (3.1) and (3.2) have the solutions $y(x)=x^{3} \ln x$ and $y(x)=\operatorname{arccot} x$. In table $\mathrm{I}$ we give the experimental results for the error and the order of the numerical solution NS(2), which uses the trapezoidal rule (2), of equations (3.1)-left and (3.2)-right on the interval $[0,1]$. The rest of the paper is organized as follows. In section two we construct the numerical solution of first order ODEs by transforming them to integral equations in the form (3) and we also construct the numerical solutions which use the corresponding shifted quadrature formulas. In section three of the paper we apply the method for numerical solution of second order ordinary differential equations (ODEs) by converting them to Voltera integral equations
TABLE I

\begin{tabular}{|l|cc|cc|}
\hline \multicolumn{1}{|c|}{$h$} & Error & Order & Error & Order \\
\hline 0.005 & $4.545 \times 10^{-6}$ & 2.000 & $2.802 \times 10^{-6}$ & 2.000 \\
0.0025 & $1.136 \times 10^{-6}$ & 2.000 & $7.006 \times 10^{-7}$ & 2.000 \\
0.00125 & $2.841 \times 10^{-7}$ & 2.000 & $1.751 \times 10^{-7}$ & 2.000 \\
\hline
\end{tabular}

\section{NUMERICAL SOLUTION OF FIRST ORDER ODES}

In this section we construct the numerical solution of first order ODEs by first transforming them to integral equations which are solved with Nyström method. In [2] we obtain the second order approximations

$$
\begin{aligned}
& \frac{h}{2} \sum_{k=0}^{N} \bar{E}_{k} y_{N-k}=\int_{a}^{b} y(x) d x+O\left(h^{2}\right), \\
& \frac{h}{2} \sum_{k=0}^{N} \bar{B}_{k} y_{N-k}=\int_{a}^{b} y(x) d x+O\left(h^{2}\right),
\end{aligned}
$$

where $\bar{E}_{0}=(\pi-1) / 2, \bar{B}_{0}=\left(\pi^{2}-6\right) / 4$ and the rest of the weights are defined with (1). The two approximations (4) and (5) require that the integrand function satisfies $y(a)=0$. We use the method (see [3], [7]) for extending the approximations to the class of all differentiable functions by changing the values of the last weights. By applying approximation (4) to the function $y(x)-y(a)$ we obtain

$$
\begin{aligned}
& \frac{h}{2} \sum_{k=0}^{N-1} \bar{E}_{k}\left(y_{N-k}-y_{0}\right)=\int_{a}^{b}(y(x)-y(a)) d x+O\left(h^{2}\right), \\
& \frac{h}{2}\left(\sum_{k=0}^{N-1} \bar{E}_{k} y_{N-k}+2 N y_{0}-y_{0} \sum_{k=0}^{N-1} \bar{E}_{k}\right)=\int_{a}^{b} y(x) d x+O\left(h^{2}\right) .
\end{aligned}
$$

Define $\bar{E}_{N}=2 N-\sum_{k=0}^{N-1} \bar{E}_{k}$. Therefore approximation (4) has a second-order accuracy for all differentiable functions. Similarly, when $\bar{B}_{N}=2 N-\sum_{k=0}^{N-1} \bar{B}_{k}$ approximation (5) holds for all differentiable functions in $[a, b]$. Now we apply approximations (4) and (5) for numerical solution of ODEs. Consider the first order linear ODE

$$
y^{\prime}+a y=f(x), y(0)=y_{0} .
$$

By applying integration on both sides of equation (6) we get

$$
\begin{aligned}
\int_{0}^{x} y^{\prime}(t) d t+a \int_{0}^{x} y(t) d t & =\int_{0}^{x} f(t) d t \\
y(x)+a \int_{0}^{x} y(t) d t & =F(x)
\end{aligned}
$$

where $F(x)=y_{0}+\int_{0}^{x} y(t) d t$. Equation (7) is equivalent to (6) and it is a Voltera integral equation of the second kind with a kernel $K(s)=a$. Denote by (7.1) the equation which corresponds to (7) when $a=2$ and $F_{1}(x)=x^{3}(4(2+$ $x) \ln x-x) / 8$. Equation (7.1) has the solution $y(x)=x^{3} \ln x$ and it is equivalent to equation (6) with a right hand side $f_{1}(x)=x^{2}(1+(3+2 x) \ln x)$. Denote by (7.2) the integral equation which corresponds to equation (7) with $a=3$, 
$F_{2}(x)=(1+3 x) \operatorname{arccot} x+3 \ln \left(1+x^{2}\right) / 2$ and initial condition $y(0)=y_{0}=F_{0}(0)=\pi / 2$. Equation (7.2) has the solution $y(x)=\arccos x$ and it is equivalent to equation (6) with a right hand side $f_{2}(x)=3 \operatorname{arccot} x-1 /\left(1+x^{2}\right)$. Equations (7.1) and (7.2) are integral equations in the form (3) and can be solved numerically with methods NS(4) and NS(5), which use quadrature formulas (4) and (5). In table II we give the experimental results for the error and the order of the numerical solution NS(4) of equations (7.2) and (7.3).

TABLE II

\begin{tabular}{|l|cc|cc|}
\hline \multicolumn{1}{|c|}{$h$} & Error & Order & Error & Order \\
\hline 0.005 & $7.202 \times 10^{-6}$ & 1.995 & $2.918 \times 10^{-6}$ & 1.969 \\
0.0025 & $1.804 \times 10^{-6}$ & 1.997 & $7.412 \times 10^{-7}$ & 1.977 \\
0.00125 & $4.513 \times 10^{-7}$ & 1.999 & $1.870 \times 10^{-7}$ & 1.987 \\
\hline
\end{tabular}

The results for the error and order of the numerical solution NS(5) of equations (7.2) and (7.3) are given in table III.

TABLE III

\begin{tabular}{|l|cc|cc|}
\hline \multicolumn{1}{|c|}{$h$} & Error & Order & Error & Order \\
\hline 0.005 & $3.823 \times 10^{-6}$ & 2.004 & $1.351 \times 10^{-6}$ & 1.996 \\
0.0025 & $9.544 \times 10^{-7}$ & 2.002 & $3.385 \times 10^{-7}$ & 1.997 \\
0.00125 & $2.384 \times 10^{-7}$ & 2.001 & $8.476 \times 10^{-8}$ & 1.998 \\
\hline
\end{tabular}

Now we construct the shifted quadratures which correspond to formulas (4) and (5). From [2]

$$
\begin{aligned}
& \frac{h}{2} \sum_{k=0}^{N} \bar{E}_{k} y_{N-k}=\int_{a}^{b} y(x) d x+\frac{1}{4} f(b) h+O\left(h^{2}\right), \\
& \frac{h}{2} \sum_{k=0}^{N} \bar{B}_{k} y_{N-k}=\int_{a}^{b} y(x) d x+\frac{3}{4} f(b) h+O\left(h^{2}\right),
\end{aligned}
$$

where the weights $\bar{E}_{k}$ and $\bar{B}_{k}$ are defined with (1) for all indices $k=0,1, \cdots, n$. From the mean value theorem we have the second order approximation

$$
\int_{a}^{b+c h} y(x) d x=\int_{a}^{b} y(x) d x+c f(b) h+O\left(h^{2}\right) .
$$

From (8) and (10)

$$
\frac{h}{2} \sum_{k=0}^{N} \bar{E}_{k} y_{N-k}=\int_{a}^{b+h / 4} y(x) d x+O\left(h^{2}\right) .
$$

Shifted quadature (11) has a requirement that the integrand function satisfies the condition $y(a)=0$. By applying (11) to the function $y(x)-y(a)$ we find

$\frac{h}{2} \sum_{k=0}^{N-1} \bar{E}_{k}\left(y_{N-k}-y_{0}\right)=\int_{a}^{b+h / 4}(y(x)-y(a)) d x+O\left(h^{2}\right)$,

Shifted quadrature (11) has a second order accuracy for all differentiable functions when the weight $\bar{E}_{N}$ is defined as

$$
\bar{E}_{N}=\frac{2}{h}\left(b-a+\frac{h}{4}\right)-\sum_{k=0}^{N-1} \bar{E}_{k}=2 N+\frac{1}{2}-\sum_{k=0}^{N-1} \bar{E}_{k}
$$

Similarly from (9) and (10) we obtain

$$
\frac{h}{2} \sum_{k=0}^{N} \bar{B}_{k} y_{N-k}=\int_{a}^{b+3 h / 4} y(x) d x+O\left(h^{2}\right) .
$$

Shifted quadrature (12) has a second-order accuracy when

$$
\bar{B}_{N}=2 N+\frac{3}{2}-\sum_{k=0}^{N-1} \bar{B}_{k}
$$

and the weights $\bar{B}_{0}, \bar{B}_{1}, \cdots, \bar{B}_{N-1}$ are defined with (1). The first weights $\bar{E}_{0}=\pi / 2$ and $\bar{B}_{0}=\pi^{2} / 4$. Now we construct the numerical solution of integral equation (7) which uses shifted quadrature (11). By approximating the definite integral in (7) at the point $x_{n+1 / 4}$ with (11) we obtain

$$
y_{n+1 / 4}+\frac{1}{2} a h \sum_{k=0}^{n-1} \bar{E}_{k} y_{n-k}=F_{n+1 / 4}+O\left(h^{2}\right) .
$$

Let $\left\{u_{n}\right\}_{n=0}^{N}$ be the numerical solution of (7). From the second order approximation

$$
y_{n+1 / 4}=\frac{5 y_{n}-y_{n-1}}{4}+O\left(h^{2}\right)
$$

we obtain the recursive formula for the numerical solution

$$
u_{n}=\frac{1}{5+\pi a h}\left(4 F_{n+1 / 4}+u_{n-1}-2 a h \sum_{k=1}^{n-1} \bar{E}_{k} y_{n-k}\right) \text {, }
$$

with an initial condition $u_{0}=F_{0}$. In table IV we give the experimental results for the error and the order of the numerical solution NS1 of equations (7.2)-left and (7.3)-right.

TABLE IV

\begin{tabular}{|l|cc|cc|}
\hline \multicolumn{1}{|c|}{$h$} & Error & Order & Error & Order \\
\hline 0.005 & $5.638 \times 10^{-6}$ & 2.000 & $1.323 \times 10^{-6}$ & 1.996 \\
0.0025 & $1.409 \times 10^{-6}$ & 2.000 & $3.314 \times 10^{-7}$ & 1.998 \\
0.00125 & $3.522 \times 10^{-7}$ & 2.000 & $8.291 \times 10^{-8}$ & 1.989 \\
\hline
\end{tabular}

The numerical solution of integral equation (7) which uses shifted quadrature (12) is obtained from the approximation of the definite integral in (7) at the point $x_{n+3 / 4}$ with (12).

$$
y_{n+3 / 4}+\frac{1}{2} a h \sum_{k=0}^{n-1} \bar{B}_{k} y_{n-k}=F_{n+3 / 4}+O\left(h^{2}\right) .
$$

From

$$
y_{n+3 / 4}=\frac{7 y_{n}-3 y_{n-1}}{4}+O\left(h^{2}\right)
$$

we obtain the recursive formula of the numerical solution

$$
u_{n}=\frac{2}{14+a \pi^{2} h}\left(4 F_{n+3 / 4}+3 u_{n-1}-2 a h \sum_{k=1}^{n-1} \bar{E}_{k} y_{n-k}\right) \text {. }
$$

The experimental results for the error and the order of the numerical solution NS2 of equations (7.2) and (7.3) are given in table V. 
TABLE V

\begin{tabular}{|l|cc|cc|}
\hline \multicolumn{1}{|c|}{$h$} & Error & Order & Error & Order \\
\hline 0.005 & $6.249 \times 10^{-5}$ & 1.990 & $1.352 \times 10^{-5}$ & 1.900 \\
0.0025 & $1.567 \times 10^{-5}$ & 1.995 & $3.532 \times 10^{-6}$ & 1.936 \\
0.00125 & $3.924 \times 10^{-6}$ & 1.997 & $9.054 \times 10^{-7}$ & 1.964 \\
\hline
\end{tabular}

\section{NUMERICAL SOLUTION OF SECOND ORDER ODES}

We apply the method from section 2 for computing the numerical solution of the second order ODE

$$
y^{\prime \prime}+3 y^{\prime}+2 y=f(x), y(0)=y_{0}, y^{\prime}(0)=y_{0}^{\prime} .
$$

Equation (13) is transformed to an integral equation in the form (3) by applying successive integration to both sides

$$
\begin{gathered}
\int_{0}^{x} y^{\prime \prime}(u) d u+3 \int_{0}^{x} y^{\prime}(u) d u+2 \int_{0}^{x} y(u) d u=\int_{0}^{x} f(u) d u, \\
y^{\prime}(x)+3 y(x)+2 \int_{0}^{x} y(u) d u=3 y_{0}+y_{0}^{\prime}+\int_{0}^{x} f(u) d u .
\end{gathered}
$$

Integrate again both sides

$$
y(x)+3 \int_{0}^{x} y(t) d t+2 \int_{0}^{x} \int_{0}^{t} f(u) d u d t=F(x),
$$

where $F(x)=y_{0}+\left(3 y_{0}+y_{0}^{\prime}\right) x+\int_{0}^{x} \int_{0}^{t} f(u) d u d t$. By changing the order of integration of the double integral we get

$\int_{0}^{x} \int_{0}^{t} f(u) d u d t=\int_{0}^{x} \int_{u}^{x} f(u) d t d u=\int_{0}^{x}(x-u) f(u) d t d u$.

Equation (14) is transformed to

$$
y(x)+\int_{0}^{x}(3+2 x-2 t) y(t) d t=F(x) .
$$

Equation (15) is a Volterra integral equation of the second kind with a kernel $K(s)=3+2 s$. In section 1 we compute the numerical solution $\mathrm{NS}(2)$ of equations (3.1) and (3.2), which are also integral equations in the form (15). In table VI we give the results for the maximal error and order of numerical solution NS(4), which uses quadrature formula (4), of equations (3.1)left and (3.2)-right and step sizes $h=0.005,0.0025,0.00125$.

TABLE VI

\begin{tabular}{|l|cc|cc|}
\hline \multicolumn{1}{|c|}{$h$} & Error & Order & Error & Order \\
\hline 0.005 & $6.730 \times 10^{-6}$ & 1.997 & $5.885 \times 10^{-6}$ & 1.947 \\
0.0025 & $1.684 \times 10^{-6}$ & 1.998 & $1.499 \times 10^{-7}$ & 1.972 \\
0.00125 & $4.213 \times 10^{-7}$ & 1.999 & $3.805 \times 10^{-7}$ & 1.978 \\
\hline
\end{tabular}

The results for the error and the order of the numerical solution $\mathrm{NS}(5)$ of equations (3.1) and (3.2) are given in table VII.

TABLE VII

\begin{tabular}{|l|cc|cc|}
\hline \multicolumn{1}{|c|}{$h$} & Error & Order & Error & Order \\
\hline 0.005 & $3.563 \times 10^{-6}$ & 2.002 & $2.795 \times 10^{-6}$ & 2.008 \\
0.0025 & $8.901 \times 10^{-7}$ & 2.001 & $6.969 \times 10^{-7}$ & 2.004 \\
0.00125 & $2.224 \times 10^{-7}$ & 2.000 & $1.740 \times 10^{-7}$ & 2.001 \\
\hline
\end{tabular}

\section{CONCLUSION}

In the paper we construct second-order numerical solutions of ordinary differential equations by converting them to integral equations and applying the quadrature formulas from [2] . Numerical solutions NS(4) and NS(5) involve additional multiplications compared to the standard method NS(2) and have a longer computational time. One advantage of the methods discussed in the paper is that the linear ODEs are equivalent to Volterra integral equations in the form (3) and they can be solved numerically with $\mathrm{NS}(*)$ which uses an appropriate quadrature. All methods discussed in the paper involve $O\left(N^{2}\right)$ computations and have comparable performance. From the experiments presented in the paper and the results of additional experiments we can conclude that the numerical solutions discussed in the paper have a stable and efficient performance. In future work we will prove the convergence of the numerical solutions constructed in the paper and we will apply the methods for numerical solution of other classes of ordinary differential equations.

\section{REFERENCES}

[1] Y. Dimitrov, "Approximations for the Caputo derivative (I)," Journal of Fractional Calculus and Applications, vol. 9 no. 1, 2018, pp. 35-63, http://fcag-egypt.com/Journals/JFCA/.

[2] Y. Dimitrov, R. Miryanov and V. Todorov, "Quadrature formulas and Taylor series of secant and tangent", Economics and computer science, vol. 4, 2017, pp. 23-40.

[3] Y. Dimitrov, R. Miryanov and V. Todorov, "Asymptotic expansions and approximations of the Caputo derivative", Computational and Applied Mathematics, vol. 37 no. 4, 2018, pp. 5476-5499, https://doi.org/10.1007/s40314-018-0641-3.

[4] H. Ding and C. Li, "High-order numerical algorithms for Riesz derivatives via constructing new generating functions", Journal of Scientific Computing, vol. 71 no. 3, 2017, pp. 759-784, https://doi.org/10.1007/s10915-016-0317-3.

[5] G. H. Gao, Z. Z. Sun and H. W. Zhang, "A new fractional numerical differentiation formula to approximate the Caputo fractional derivative and its applications", Journal of Computational Physics, vol. 259, 2014, pp. 33-50, https://doi.org/10.1016/j.jcp.2013.11.017.

[6] E. J. Nyström, "Über die praktische Auflösung von Integralgleichungen mit Anwendungen auf Randwertaufgaben", Acta Mathematica, vol. 54 no. 1, 1930, pp. 185-204, https://doi.org/10.1007/BF02547521.

[7] V. Todorov, Y. Dimitrov and I. Dimov, "Second order shifted approximations for the first derivative", Studies in Computational Intelligence, Proc. Inter. Conf. HPC 2019, Borovetz, Bulgaria, 2019, in press.

[8] R. Farnoosh and M. Ebrahimi, "Monte Carlo method for solving Fredholm integral equations of the second kind", Applied Mathematics and Computation, vol. 195, 2008, pp. 309-315, https://doi.org/10.1016/j.amc.2007.04.097.

[9] S. Kim, "Solving singular integral equations using Gaussian quadrature and overdetermined system", Computers \& Mathematics with Applications, vol. 35 no. 10, 1998, pp. 63-71, https://doi.org/10.1016/S08981221(98)00073-X.

[10] Y. Zaizai and H. Zhimin, "Using the Monte Carlo method to solve integral equations using a modified control variate", $A p$ plied Mathematics and Computation, vol. 242, 2014, pp. 764-777, https://doi.org/10.1016/j.amc.2014.06.079. 